\title{
Optimal IIR Filter Design using Differential Evolution Algorithm
}

\author{
Navdeep Goel \\ Assistant Professor \\ YCoE, Talwandi Sabo
}

\author{
Sukhmanpreet Singh \\ Assistant Professor \\ RBIEBT Kharar
}

\begin{abstract}
Digital filter is mathematical algorithm that operates on discrete time signals. Different optimization algorithms can be utilized to determine the impulse response of coefficient of such a filter. Optimization problems for the design of digital filters are often complex, highly nonlinear, and multimodal in nature. The problems usually exhibit many local minima. Ideally, the optimization method should lead to the global optimum of the objective function with minimum amount of computation. Classical optimization methods are generally fast and efficient, and have been found to work reasonably well for the design of digital filters. These methods are very good in locating local minima. Therefore, they tend to locate minima in the locale of the initialization point. In recent years, a variety of algorithms have been proposed for global optimization including stochastic or heuristic algorithms; one such technique is Differential Evolution (DE). This paper presents an efficient DE based optimization technique for designing digital IIR filter by solving constrained multivariable optimization problem, to optimize the magnitude response of digital filters employing stability constraints using DE with opposition based strategy.
\end{abstract}

\section{Keywords}

IIR Filter design, magnitude error (norm approximation error), Differential evolution Algorithm.

\section{INTRODUCTION}

Digital filter design is always an important issue in digital signal processing. Digital filter can be designed by using the values of the past outputs and present inputs, an operation brought about by convolution. If such a filter is subjected to an impulse then its output need not necessarily become zero. The impulse response of such a filter can be infinite in duration. This indicates that system is prone to feedback and instability. In digital IIR filter design, there are principally two approaches [14], namely:

\section{- Transformation approach}

- Optimization approach.

The approach to the design of digital IIR filter involves the transformation of an analog filter into a digital filter at a given set of prescribed specifications. In general, a bilinear transformation or impulse invariance is adopted in the transformation approach [1]. Impulse invariance procedure involve the choosing the response of the digital filter as an equispaced sampled version of the analog filter various steps involved in designing analog filter from impulse invariance are

- $\quad$ Decide upon the desired frequency response
- Design an appropriate analog filter

- Calculate the Impulse response of this filter

- Sample the value of analog filter impulse response

- Use the result as a filter coefficients

The Bilinear transformation is a mathematical relation by which an s- domain equation is converted directly in to the $\mathrm{z}$ domain.

But the performance of digital IIR filter designed by using the transformation approach is not up to the mark in most cases. In the optimization approach, with some criteria, various optimization methods have been proposed to obtain optimal filter performances to some extent, where the norm-error, mean-square-error, and ripple magnitudes (tolerances) of both pass band and stop band are usually used as criteria to measure the performance of the designed digital IIR filter[7,6.4. For the design of a digital IIR filter, the following constraints need to be strictly imposed in order that the overall design criteria are met in a satisfactory fashion:

- Determination of the lowest filter order.

- Filter stability.

- Fulfillment of the tolerance settings that are determined by minimizing the ripple magnitudes of both pass band and stop band in the optimization procedure.

These constraints always pose great difficulty in the process of optimization. So, it is very necessary to develop efficient optimization algorithms to deal with digital IIR filter design problems.

\section{PROBLEM STATEMENT}

Digital filter design problem involves the determination of a set of filter coefficients which meet performance specifications such as pass-band width and corresponding gain, width of the stop-band and attenuation, band edge frequencies and tolerable peak ripple in the pass band and stop-band. The transfer function of IIR is represented by cascading first and second order sections to avoid the coefficient quantization problem which causes instability [4, 8]. In cascade realization coefficient range is limited. The structure of cascading type digital IIR filter is shown in (2.1):

$$
H(z)=A \prod_{i=1}^{M}\left[\frac{\left(a_{i}+z\right)}{\left(b_{i}+z\right)}\right] \times \prod_{j=1}^{N} \frac{q_{j}+p_{j} z+z^{2}}{s_{j}+r_{j}+z^{2}}
$$

where, $a_{i}, b_{i}, q_{j}, r_{j}, s_{j}$ are filter coefficient

$\mathrm{V}$ is the number of filter coefficients $(V=1+2 M+4 N)$ 
Substitute $\quad z=e^{j \omega}$ in above (2.1)

$$
H(z)=A \prod_{i=1}^{M} \frac{1+a_{i} e^{-j \omega}}{1+b_{i} e^{j \omega}} \times \prod_{j=1}^{N} \frac{1+p_{j} z^{-1}+q_{j}+e^{-2 j \omega}}{1+r_{j} e^{-j \omega}+s_{j} e^{-2 j \omega}}
$$

Euler's identities are given below

$$
\begin{aligned}
& e^{j \omega}=\cos \omega+j \sin \omega \\
& e^{-j \omega}=\cos \omega-j \sin \omega
\end{aligned}
$$

(2.2) is rewritten below considering (2.3) and (2.4) becomes

$$
H(z)=A \prod_{i=1}^{M} \prod_{j=1}^{N} \frac{1+a_{i}(\cos \omega-j \sin \omega)}{1+b_{i}(\cos \omega-j \sin \omega)} \times
$$

Rewriting above (2.5)

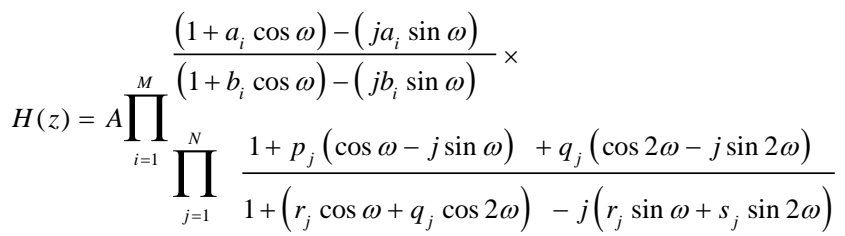

Magnitude of transfer function is obtained as:

$$
\begin{aligned}
& |H(z)|=A \prod_{i=1}^{M} \frac{\sqrt{\left(1+a_{i} \cos \omega\right)^{2}+\left(a_{i} \sin \omega\right)^{2}}}{\sqrt{\left(1+b_{i} \cos \omega\right)^{2}+\left(b_{i} \sin \omega\right)^{2}}} \times \\
& \prod_{j=1}^{N} \frac{\sqrt{\left(1+p_{j} \cos r \omega+q_{j} \cos 2 \omega\right)^{2}+\left(p_{j} \sin \omega+q_{j} \sin 2 \omega\right)^{2}}}{\sqrt{\left(1+r_{j} \cos \omega+s_{j} \cos 2 \omega\right)^{2}+\left(r_{j} \sin \omega+s_{j} \sin 2 \omega\right)^{2}}}
\end{aligned}
$$

Magnitude of transfer function on simplification is rewritten

$$
\begin{aligned}
& |H(z)|=A \frac{\sqrt{1+a^{2}+2 a_{1} \cos \omega}}{\sqrt{1+b^{2}{ }_{1}+b_{1} \cos \omega}} \times \\
& \frac{\sqrt{1+p^{2}{ }_{1}+q_{1}^{2}+2 q_{1}\left(2 \cos ^{2} \omega-1\right)+2 p_{1}\left(1+q_{1}\right) \cos \omega}}{\sqrt{1+r_{1}^{2}+s_{1}^{2}+2 s_{1}\left(2 \cos ^{2} \omega-1\right)+2 r_{1}\left(1+s_{1}\right) \cos \omega}}
\end{aligned}
$$

where, $\omega=\frac{\Pi}{K} n$

Order of filter is given by $O=M+2 N$, where $\mathrm{M}$ and $\mathrm{N}$ are first order and second order poles and zero. In the IIR filter, the coefficients are optimized such that the approximation error function for magnitude is to be minimized. The magnitude response is specified at $K$ equally spaced discrete frequency points in pass-band and stop-band. $e(x)$ Denotes the absolute error and is defined as below:

$$
e(x)=\sum_{n=0}^{K}\left|H_{d}\left(\omega_{i}\right)-\right| H(\omega) \mid
$$

Desired magnitude response, $H_{d}\left(\omega_{i}\right)$ of IIR filter is given

$$
H_{d}\left(\omega_{i}\right)= \begin{cases}1, & ; \text { for } \quad \omega_{i} \in \text { passband } \\ 0, & ; \text { for } \quad \omega_{i} \in \text { stopband }\end{cases}
$$

The ripple magnitudes of pass-band and stop-band are given by $\delta_{1}(x)$ and $\delta_{2}(x)$, respectively [2]. Ripple magnitudes are defined as:

$$
\begin{aligned}
& \delta_{1}(x)=\max _{i}\left\{\left|H\left(\omega_{i}, x\right)\right|\right\}-\underset{\omega_{i}}{\min }\left\{\left|H\left(\omega_{i}, x\right)\right|\right\} \\
& ; \omega_{i} \in \text { passband }
\end{aligned}
$$

and

$$
\delta_{2}(x)=\underset{\omega_{i}}{\max }\left\{\left|H\left(\omega_{i}, x\right)\right|\right\} ; \omega_{i} \in \text { stopband }
$$

Stability constraints are included in the design of casual recursive filters, which are obtained by using the Jury method [5]. The multivariable constrained optimization problem is stated as below:

Minimize $f(x)=e(x)$

Subject to the stability constraints:-

$$
\begin{aligned}
& 1+b_{i} \geq 0(i=1,2, \ldots ., M) \\
& 1-b_{i} \geq 0(i=1,2, \ldots ., M) \\
& 1-s_{j} \geq 0(j=1,2, \ldots ., N) \\
& 1+r_{j}+s_{j} \geq 0(j=1,2, \ldots ., N) \\
& 1-r_{j}+s_{j} \geq 0(j=1,2, \ldots ., N)
\end{aligned}
$$

Scalar constrained optimization problem is converted in to unconstrained multivariable optimization problem using penalty method. Augmented function is defined as in (2.20)

$$
A(x)=e(x)+r\left(P_{\text {term }}\right)
$$

where,

$$
\begin{aligned}
& P_{\text {term }}=\sum_{i=1}^{M}\left\langle 1+b_{i}\right\rangle^{2}+\sum_{i=1}^{M}\left\langle 1-b_{i}\right\rangle^{2}+\sum_{j=1}^{N}\left\langle 1-s_{j}\right\rangle^{2} \\
& +\sum_{j=1}^{N}\left\langle 1+r_{j}+s_{j}\right\rangle^{2}+\sum_{j=1}^{N}\left\langle 1-r_{j}+s_{j}\right\rangle^{2}
\end{aligned}
$$

with $\mathrm{r}$ is a penalty parameter having large value.

Bracket function for constraint is given by. (2.21) is given below:- 


$$
\left\langle 1+b_{i}\right\rangle= \begin{cases}\left(1+b_{i}\right)^{2} & ; \text { if }\left(1+b_{i}\right)<0 \\ 0 & ; \text { if }\left(1+b_{i}\right) \geq 0\end{cases}
$$

Similarly bracket functions for other constraints given by (2.22) are taken

\section{DIFFERENTIAL EVOLUTION APPROACH FOR DESIGN OF IIR DIGITAL FILTERS}

$\mathrm{DE}$ is a population-based stochastic function minimizes relating to evolutionary computation, whose simple features make it very attractive for numerical optimization[8]. DE uses a rather greedy and less stochastic approach to problem solving than do evolutionary algorithms. DE combines simple arithmetical operators with the classical operators of recombination, mutation, and selection to evolve from a randomly generated starting population to a final solution. The DE algorithm was first introduced by Storn and Price in 1995 and was successfully applied in the optimization of some well-known nonlinear, non-differentiable, and nonconvex functions by Storn [3]. The different variants of DE are classified using the following notation: $\mathrm{DE} / \mathrm{z} / \mathrm{y} / \mathrm{x}$, where $\mathrm{z}$ indicates the method for selecting the parent chromosome that will form the base of the mutated vector, $y$ indicates the number of difference vectors used to perturb the base chromosome, and $\mathrm{x}$ indicates the recombination mechanism used to create the offspring population. The bin acronym indicates that the recombination is controlled by a series of independent binomial experiments. The fundamental idea behind $\mathrm{DE}$ is a scheme whereby it generates the trial parameter vectors. In each step, the DE mutates vectors by adding weighted, random vector differentials to them. If the cost of the trial vector is better than that of the target, the trial vector replaces the target vector in the next generation $[11,12$, 13]. The variant implemented here was the $D E / \mathrm{rand} / 3 / \mathrm{bin}$, which involved the following steps and procedures [3].

\subsection{Parameter setup}

The user must choose the key parameters that control the Differential Evolution, i.e. population size (L), boundary constraints of optimization variables (V), mutation factor $\left(f_{m}\right)$, crossover rate (CR), and the stopping criterion of maximum number of iterations (generations) $t_{\max }$.

The set of filter coefficients (X) of IIR digital filter is represented as the population. For a system with $V$ generators, the population is represented as a vector of length $V$. If there are $\mathrm{L}$ members in the population, the complete population is represented as a matrix as below:

Population $=\left[\begin{array}{ccccc}x_{11}^{t} & x_{12}^{t} & . . & . . & x_{1 V}^{t} \\ x_{21}^{t} & x_{22}^{t} & . . & . . & x_{2 V}^{t} \\ . & . & x_{i j}^{t} & . . & . \\ . & . & . . & . . & . \\ x_{L 1}^{t} & x_{L 2}^{t} & . . & . . & x_{L V}^{t}\end{array}\right]$

where, $x_{i j}^{t}$ the jth element of $\mathrm{V}$ is set of committed decision variables giving ith individual of a population. In other words it represents the filter coefficients of IIR digital filter $j$ of the possible solution $i$. Further, $X_{i}^{t}=\left[x_{i 1}^{t}, x_{i 2}^{t}, \ldots, x_{i V}^{t}\right]^{T}$ stands for the position of the ith individual of a population of real valued $V$-dimensional vectors;

\subsection{Initialization of an individual population}

Set generation $\mathrm{t}=0$. Initialize a population $x_{i j}^{t}(\mathrm{j}=1,2, \ldots$, $\mathrm{V} ; \mathrm{i}=1,2, \ldots, \mathrm{L}$ ) individuals (real valued $\mathrm{V}$-dimensional solution vectors) with random values generated according to a uniform probability distribution in the $\mathrm{V}$-dimensional problem space. Initialize the entire solution vector population within the given upper and lower limits of the search space.

$x_{i j}^{t}=x_{i}^{\min }+\operatorname{rand}()\left(x_{i}^{\max }-x_{i}^{\min }\right)$

$(\mathrm{j}=1,2, \ldots, \mathrm{V} ; \mathrm{i}=1,2, \ldots, \mathrm{L})$

where, $x_{j}^{\min }$ and $x_{j}^{\max }$ are lower and upper limits of filter coefficients.

\subsection{Opposition-based learning: a} concept

Evolutionary optimizations methods start with some initial solutions (initial population) and try to improve them toward some optimal solution(s). The process of searching terminates when some predefined criteria are satisfied. In the absence of a priori information about the solution, we usually start with random guesses [8]. The computation time, among others, is related to the distance of these initial guesses from the optimal solution. We can improve our chance of starting with a closer (fitter) solution by simultaneously checking the opposite solution [6]. By doing this, the fitter one (guess or opposite guess) can be chosen as an initial solution. In fact, according to the theory of probability, $50 \%$ of the time a guess is further from the solution than its opposite guess [6]. Therefore, starting with the closer of the two guesses (as judged by its fitness) has the potential to accelerate convergence. The same approach can be applied not only to initial solutions but also continuously to each solution in the current population $[6,14$, $8,9]$.

$$
\begin{gathered}
x_{i+L, j}^{t}=x_{j}^{\min }+x_{j}^{\max }-x_{i j}^{t} \\
(\mathrm{j}=1,2, \ldots, \mathrm{S} ; \mathrm{i}=1,2, \ldots, \mathrm{L})
\end{gathered}
$$

where, $x_{j}^{\min }$ and $x_{j}^{\max }$ are lower and upper limits of filter coefficients.

\subsection{Evaluation of the individual population}

The goal is to minimize the objective function. The elements of parent/offspring $x_{i j}^{t}$ may violate constraint. This violation is corrected by fixing them either at lower or upper limits [8] as described in (3.1.29):

$$
x_{i j}^{t}=\left\{\begin{array}{l}
x_{i}^{\min } ; x_{i j}^{t}<x_{i}^{\min } \\
x_{i}^{\max } ; x_{i j}^{t}>x_{i}^{\max } \\
x_{i j}^{t} ; x_{i}^{\min } \leq x_{i j}^{t} \leq x_{i}^{\max }
\end{array} \quad(\mathrm{i}=1,2, \ldots, \mathrm{V} ; \mathrm{j}=1,2,, \mathrm{~L})\right.
$$

A penalty term is introduced in the objective function to penalize its fitness value. when so introduced, objective function is changed to the following generalized form: 


$$
A^{j}=e\left(x_{i j}^{t}\right)+\phi^{j} \quad(\mathrm{j}=1,2, \ldots, \mathrm{L})
$$

where,

Penalty factor is given by

$$
\phi^{j}= \begin{cases}\left(x_{i j}^{t}-x_{i}^{\min }\right)^{2} & ; x_{i j}^{t}<x_{i}^{\min } \\ \left(x_{i}^{\max }-x_{i j}^{t}\right)^{2} & ; x_{i j}^{t}>x_{i}^{\max } \\ 0 & ; x_{i}^{\min } \leq x_{i j}^{t} \leq x_{i}^{\max }\end{cases}
$$

\subsection{Mutation operation (Differential \\ Operation)}

Mutation is an operation that adds a vector differential to a population vector of individuals. Two difference vectors are used as a perturbation in this mutation strategy according to the following equation:

$$
\begin{aligned}
& Z_{i j}^{t}=x_{i j}^{t}+f_{m}\left(x_{b j}^{t}-x_{i j}^{t}\right)+ \\
& f_{m}\left(x_{r 1 j}^{t}-x_{r 2 j}^{t}\right)+f_{m}\left(x_{r 3 j}^{t}-x_{r 4 j}^{t}\right) \\
& (j=1,2, \ldots, V ; i=1,2, \ldots, L)
\end{aligned}
$$

$f_{m}(t)$ is the mutation factor and $f_{m}(t)>0$ is a real parameter, which controls the amplification of the difference between two individuals with indexes $R_{2}$ and $R_{3}$ so as to avoid search stagnation and is usually a constant value taken from the range $[0.4,1]$

The mutation operation using the difference between two randomly selected individuals causes the mutant individual to escape from the search domain. If an optimized variable for the mutant individual is outside of the domain search, then this variable is replaced by its lower bound or its upper bound so that each individual can be restricted to remain within the search domain [8].

\subsection{Recombination operation}

After the mutation operation, recombination is applied to the population. Recombination is used to get a trial vector by replacing certain parameters of the target vector by the corresponding parameters of a randomly generated donor vector. For each vector, $Z_{i}^{t+1}$, an index R5(i) $\in\{1,2, \ldots$, $\mathrm{V \}}$ is randomly chosen using a uniform distribution, and a trial vector, $U_{i}^{t+1}=\left[U_{i 1}^{t+1}, U_{i 2}^{t+1}, \ldots, U_{i V G}^{t+1}\right]^{T}$

$U_{i j}^{t+1}=\left\{\begin{array}{ll}Z_{i j}^{t} & \text { if }(R 4(j) \leq C R) \text { or }(j=R 5(i)) \\ x_{i j}^{t} & \text { if }(R 4(j)>C R) \text { or }(j \neq R 5(i))\end{array}(\mathrm{j}=1, \quad 2\right.$, $\ldots, \mathrm{V} ; \mathrm{I}=1,2, \ldots, \mathrm{L})$

Usually, the performance of a DE algorithm depends on three variables: the population size, the mutation factor $f_{m}(t)$ and the $\mathrm{CR}$.

\subsection{Selection operation}

Selection is the process whereby better offspring are produced. To decide whether the vector $U_{i}^{t+1}$ should be a member of the population comprising the next generation, it is compared with the corresponding vector $X_{i}^{t}$. Thus, if $f$ denotes the cost function under minimization, then

$$
\begin{aligned}
& x_{i j}^{t+1}=\left\{\begin{array}{ll}
U_{i j}^{t+1}(j=1,2, . ., V) & ; \text { if } A\left(U_{i}^{t+1}\right)<A\left(X_{i}^{t}\right) \\
x_{i j}^{t} \quad(j=1,2, . ., V) & ; \text { otherwise }
\end{array} \quad(\mathrm{i}=\right. \\
& 1,2, \ldots, \mathrm{L})
\end{aligned}
$$

In this case, the cost of each trial vector $U_{i}^{t+1}$ is compared with that of its parent target vector $X_{i}^{t}$. If the objective function, $\mathrm{F}$, of the target vector $X_{i}^{t}$ is lower than that of the trial vector, the target is allowed to advance to the next generation. Otherwise, a trial vector replaces the target vector in the next generation.

\subsection{Verification of the stopping}

\section{criterion}

Set the generation number for $\mathrm{t}=\mathrm{t}+1$. Proceed to Step until a stopping criterion is met, usually a maximum number of iterations (generations), $t_{\max }$. The stopping criterion depends on the type of problem.

\subsection{Flowchart for IIR filter design}

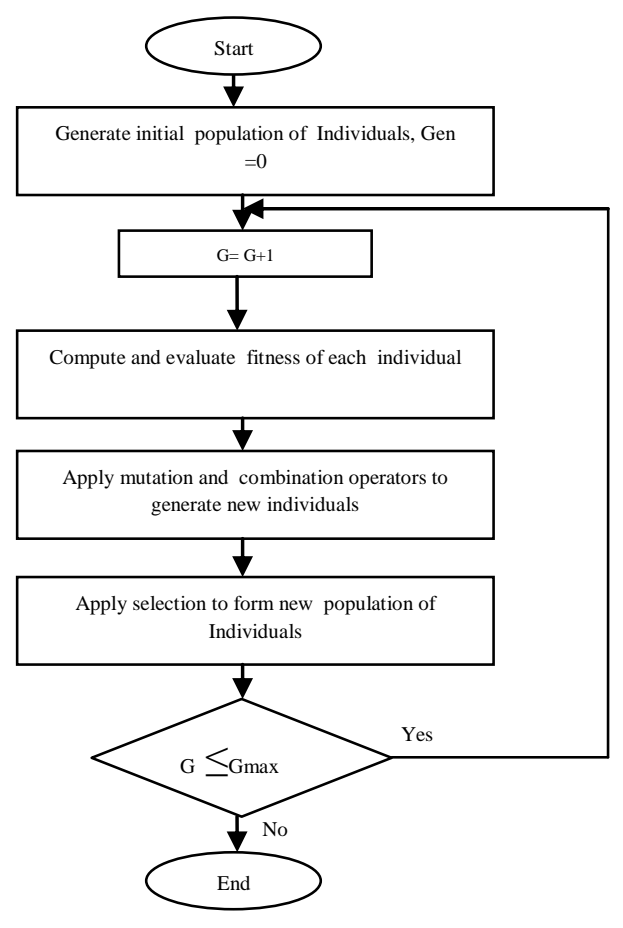

(3.1.33)

\section{RESULTS AND COMPARISON}

The computational experiments show that the proposed DE method is superior or at least comparable to other algorithms and can be efficiently applied for higher filter design. The order of the digital IIR filter is set 8 for the LP, HP, BP, and BS filters. The objective of designing the digital IIR filter is to minimize the objective function. To design digital IIR filter, 200 equally spaced points are set within the frequency 
domain $[0, \pi]$, such that the number of discrete frequency points in .(3.1.13), comes out 182 for the LP and HP filters, and 143 for the BP and BS filters along with prescribed passband and stop-band frequency range is given in Table-4.1 . The design of cascaded digital IIR filter has been implemented by evaluating filter coefficients using differential evolution. Low pass (LP), high pass (HP), band pass (BP) and band stop (BS) filters have been considered for the design.

Design conditions for these filters are given in Table-4.1.

\begin{tabular}{|c|c|c|c|}
\hline $\begin{array}{c}\text { Filter } \\
\text { Type }\end{array}$ & $\begin{array}{c}\text { Pass- } \\
\text { band }\end{array}$ & Stop-band & $|H(\omega, x)|$ \\
\hline Low-pass & $0 \leq \omega \leq 0.2$ & $0.3 \pi \leq \omega \leq \pi$ & 1 \\
\hline High-pass & $0.8 \pi \leq \omega \leq$ & $0 \leq \omega \leq 0.7 \pi$ & 1 \\
\hline Band-pass & $0.4 \pi \leq \omega \leq$ & $\begin{array}{c}0 \leq \omega \leq 0.25 \pi \\
0.75 \leq \omega \leq \pi\end{array}$ & 1 \\
\hline Band-stop & $\begin{array}{c}0 \leq \omega \leq 0.2 \\
0.75 \leq \omega \leq\end{array}$ & $0.4 \pi \leq \omega \leq 0.6$ & 1 \\
\hline
\end{tabular}

Design results are shown in Table-4.2.

\begin{tabular}{|c|c|c|c|}
\hline Method & $\begin{array}{l}\text { Magnitu } \\
\text { de error }\end{array}$ & $\begin{array}{l}\text { Pass band } \\
\text { performance }\end{array}$ & $\begin{array}{c}\text { Stop band } \\
\text { performance }\end{array}$ \\
\hline Low-pass & 1.19293 & $\begin{array}{l}.91772 \leq \mid H\left(e^{j ø}\right. \\
(.097965)\end{array}$ & $\begin{array}{l}\left|H\left(e^{j \omega}\right)\right| \leq 0 . \\
(0.076412)\end{array}$ \\
\hline High-pass & 1.860575 & $\begin{array}{l}0.96559 \leq \mid H(e \\
(0.039051)\end{array}$ & $\begin{array}{l}\left|H\left(e^{j \omega}\right)\right| \leq 0 . \\
(0.4431)\end{array}$ \\
\hline Band-pass & 0.393986 & $\begin{array}{l}0.98582 \leq \mid H(e \\
(0.20403)\end{array}$ & $\begin{array}{l}\left|H\left(e^{j \omega}\right)\right| \leq 0 . \\
(0.023780)\end{array}$ \\
\hline Band-stop & 1.605629 & $\begin{array}{l}0.91372 \leq \mid H(e \\
(0.90841)\end{array}$ & $\begin{array}{l}\left|H\left(e^{j \omega}\right)\right| \leq 0 . \\
(0.158908)\end{array}$ \\
\hline
\end{tabular}

In the computational experiments of our proposed DE (Opposition Based) approach, the following evolutionary environments are used for any type of filter: the population size is 100 , the crossover rate is 0.85 , the mutation rate is 0.1 , and the execution of the DE (Opposition Based) approach is stopped when the smallest fitness value of the chromosomes cannot be further reduced in the successive ninety generations. For the three cases studied in this work, the 200 equally spaced points are set within the frequency domain $[0$, $\pi]$.

\section{CONCLUSIONS}

In DE algorithm, there exist many trial vector generations strategies out of which a few may be suitable for solving a particular problem. Moreover three crucial control parameters involved in differential evolution algorithm, population size, scaling factor and crossover rate, may significantly influence the optimization performance. Design result shows that magnitude response of DE opposition based approach has smooth transition band. More over magnitude error is also reduced to achieve approximate ideal specifications. Moreover magnitude ripples of pass band and stop band are minimized. As shown through simulation results, the DEOB method satisfies prescribed amplitude specifications consistently.

\section{REFERENCES}

[1] E. C. Iffeachor, Digital Signal Processing: A Practical Approach, Pearson education, New Delhi $2^{\text {nd }}$ Edition 2004.

[2] K. Deb, Optimization for Engineering Design: Algorithm and Examples, Prentice Hall of India, New Delhi $1^{\text {st }}$ Edition 2003.

[3] R. Storn, "Differential Evolution - A simple and efficient Heuristics for Global Optimization over continuous spaces"1997 Journal of global optimization, vol. 2, pp no 341-359,

[4] J.T. Tsai and J.H. Chou, "Design of Optimal Digital IIR Filters by Using an Improved Immune Algorithm", 2006. IEEE Transactions on Signal Processing, vol. 54, no. 12, pp 257-271,

[5] I. Jury, Theory and Application of the Z-Transform Method, 1964 Wiley publication, New York, $1^{\text {st }}$ Edition.

[6] J.T. Tsai, J.H. Chou and T.K. Liu, "Optimal design of digital IIR filters by using hybrid Taguchi genetic algorithm", 2006 IEEE Transactions on Industrial Electronics, vol. 53, no. 3, pp 365-377.

[7] K.S. Tang, K.F. Man, S. Kwong and Z.F. Liu, "Design and optimization of IIR filter structure using hierarchical genetic algorithms" 1998, IEEE Transactions on Industrial Electronics, vol. 45, no. 3, pp. 481-487.

[8] B. Singh J. S. Dhillon and Y. S. Brar, "A hybrid Differential Evolution method for the design of IIR Digital Filter"2013, ACEEE journal on signal and image processing, Vol.4, no.1.

[9] S. Das and P. N. Suganathan, "Differential Evolution: A survey of the state-of-the-art", 2011 IEEE Transactions on Evolutionary computation.vol.15, no. 1, pp 4-31, 2011.

[10] G. Cortelazzo and M.R. Lightener, "Simultaneous design in both magnitude and group-delay of IIR and FIR filters based on multiple criterion optimization", 1984 IEEE Transactions on Acoustics, Speech, and Signal Processing, vol. 32, no.5, pp. 949-967.

[11] N. Karabooga, "Digital IIR filter design using Differential Evolution Algorithm", 2005 EURASIP Journal on Applied Signal Processing, vol. 8, pp 12691276.

[12] K.S. Reddy, M. S. Bharath, S.K. Sahoo, S. Sinha and J.P. Reddy, "Design of low power, high performance FIR filter using modified differential evolution algorithm", 
2011 International Symposium on Electronic System Design, pp 62-66.

[13] S. Chattopadhyay, S.K.Sanyal and A. Chandra, "Optimization of Control Parameter of Differential Evolution Algorithm for Efficient Design of FIR Filter"
2010 Proceedings of 13th International Conference on Computer and Information Technology pp 23-25.

[14] C.W. Tsai, C.H. Huang.and C.L. Lin, "Structured specified IIR filter and control design using real structured genetic algorithm", 2009 Applied soft computing, pp 1285-1295. 